\title{
Session 6: Infectious diseases
}

\author{
Thursday 7 October 2004. Moderators: Roberto Burioni and Mirek Gorny
}

[09.00-09.30]

[Keynote lecture]

Human monoclonal antibodies against infectious diseases: From the bench to the clinic

Alois Lang

Berna Biotech Ltd, Berne, Switzerland

Abstract not received.

[09.30-09.50]

Cross-neutralizing activity of human monoclonal antibody depends on the recognition of a conserved GPGR V3 motif of HIV-1

Miroslaw K. Gorny ${ }^{1}$, Ping Zhong ${ }^{1,3}$, Kathy Revesz ${ }^{2}$, Barbara Volsky ${ }^{1}$, Constance Williams ${ }^{1}$, Phillipe Nyambi ${ }^{1}$ and Susan Zolla-Pazner ${ }^{1,2}$

${ }^{1}$ Department of Pathology, New York University School of Medicine, New York, NY 10016, USA

${ }^{2}$ Research Enhancement Award Program, Veterans Affairs New York Harbor Healthcare System, New York, NY 10010, USA

${ }^{3}$ Shanghai Municipal Center for Disease Control and Prevention, P.R.China

Human anti-V3 antibodies (Abs), either those induced by natural HIV-1 infection or by various candidate vaccines, have the capacity to neutralize HIV-1 viruses. Although the V3 domain is hypervariable, the majority of human anti-V3 monoclonal Abs (mAbs) are not type-specific but display intra- and inter-subtype neutralizing activity against primary isolates and T celladapted virus strains. The neutralizing activity of $\mathrm{mAb}$ 447-52D, which is the most potent and cross-reactive among human anti-V3 mAbs, depends on the recognition of the relatively conserved GPGR sequence at the tip of the V3 loop.

Human mAbs were produced via fusion of heteromyeloma cells with EBV-transformed peripheral blood mononuclear cells derived from HIV-1 infected individuals. Monoclonal Ab 447-52D neutralized 92\% of 38 primary isolates carrying the GPGR V3 motif regardless of whether the viruses belonged to subtypes $\mathrm{A}, \mathrm{B}, \mathrm{F}$ or $\mathrm{H}$; in contrast, none of 19 viruses with the GPGQ and other non-GPGR/Q sequences at the tip of the V3 loop was sensitive to $\mathrm{mAb}$ 447-52D. These data are consistent with the crystallographic resolution of a complex of the Fab fragment of mAb 447-52D with a $\mathrm{V} 3$ peptide that shows that the binding specificity of the $\mathrm{mAb}$ is due to recognition of the GPGR motif at the tip of the loop. The critical role of the Arg residue in this motif was determined using viruses pseudotyped with the envelope of primary isolate CA1 containing the GPGR motif or pseudotyped with a mutated envelope with a Gln $(\mathrm{Q})$ replacing the $\operatorname{Arg}(\mathrm{R})$ at the tip of the loop. While the wildtype pseudovirus was neutralized by mAb 447-52D, the pseudovirus carrying the point mutation was resistant to neutralization. These data illuminate the structural basis for both the breadth and specificity of a broadly neutralizing human mAb and contribute to our understanding of the epitopes recognized by Abs which protect against infection with HIV-1.

\section{[09.50-10.10]}

IgM monoclonal proliferation from $\mathrm{HCV}$-positive patients binds $\mathrm{HCV}$ proteins

V. De Re, S. De Vita, D. Sansonno, D. Gasparotto, M.P. Simula, F.A. Tucci, M. Fabris, F. Dammacco and M. Boiocchi

Division of Experimental Oncology I, Centro di Riferimento Oncologico, Aviano (PN), Italy Tel.: +390434 659672; E-mail: vdere@cro.it

Introduction: Chronic hepatitis $\mathrm{C}$ virus is the leading cause of type II mixed cryoglobulinemia, a systemic immune complex-mediated disorder characterized by B-cell proliferation. Because mixed cryoglobulinemia evolves frequently into B-cell non-Hodgkin lymphoma, chronic HCV infection has been proposed as an etiologic factor for a subset of B-cell lymphoma. 
At present, one possible mechanism for $\mathrm{MC}$ and $\mathrm{HCV}$ NHL may be the continuous specific B cell clone expansions (mainly in the liver tissue and bone marrow) following chronic HCV antigen(s) stimulation [1-4] However, although many correlative data suggest that $\mathrm{HCV}$-stimulated lymphoproliferations directly to produce cryoglobulins, a demonstration is still lacking. This study aimed at demonstrating by molecular and proteomic analyses, that the variable combinatory region of the IgM, originating the serum cryoprecipitate, and the variable combinatory region of the $\operatorname{IgR}$ expressed by the lymphoproliferative process of autologous patiens share the identical amino acid sequence, and they are, therefore, encoded by a unique clonal B-cell population. Moreover, verifying the antigenic specificity of these cells to ascertain whether IgM (and therefore $\mathrm{IgR}$ ) are specific for $\mathrm{HCV}$ was also an objective of our study.

Materials and methods: $17 \mathrm{HCV}$-infected and 7 $\mathrm{HCV}$-negative patients with a diagnosis of MC syndrome were considered. Patient evaluation, including diagnosis and laboratory values, is reported in Table 1. MC syndrome HCV-negative was associated with Sjogren'syndrome in three of them, with chronic infection by hepatitis B virus (HBs Ag and HBV DNA-positive in serum) in one, and with systemic lupus erythematosus in one, while a truly "essential" MC syndrome was present in two cases.

Genomic DNA was prepared from buffy coat from bone marrow mononuclear cells. PCR reactions for IgH genescanning technique (GS) with subsequent capillary electrophoresis on ABI PRISM 3100 and elaboration of data with 3100 GeneScan 3.7 Software were performed. The $\mathrm{VH}$ and $\mathrm{VK}$ germlines regions most similar to those derived by sequencing data were identified by sequence comparison with the International Immunogenetics Database program. The amino acid sequence derived from the VH and VK DNA sequences was determined with the translated BLAST search protein program (http://www.ncbi.nlm.nih.gov/blast/).

Monoclonal IgM was purified from serum cryoprecipate by gel filtration fractionation on a Hiload Superdex 200 h 26/60 column, followed by an IgM antibodyaffinity chromatography. Residual IgG from purified IgM solution were depleted by binding to protein $G$ sepharose. IgM heavy or light chain fragments were isolated by SDS-PAGE. In-gel trypsin digestion was performed and MALDI mass spectrometry of peptides was carried out on a Voyager-DE-PRO Biospectrometry Workstation mass spectrometer. Proteins from both heavy and light chain samples were identified from the resulting peptide masses by peptide mass fingerprinting. MASCOT Search engine was used, with 100 ppm mass tolerance error. The most probable variable immunoglobulin genes from both IgM heavy and light chains were identified by the IgBlast database.

Peptides shared between monoclonal B-cell receptors and IgMs were searched matching the masses of peptides theoretically determined by a virtual digestion of $\mathrm{VH}$ and $\mathrm{VL}$ deduced proteins and masses of peptides obtained experimentally at the MALDI TOF analysis.

To detected binding of HCV antigens commercial HCV Strip ImmunoAssay was used. Strip incorporates $\mathrm{HCV}$ antigens derived from the core region, the E2 hypervariable region, the NS3 helicase region, the NS4A, the NS4B, and the NS5A regions. Sample tested consisted of IgM purified from single cryopreciptates, as secondary $\mathrm{Ab}$, an phosphatase-labeled anti-human Ab specific for IgM isotype was used. ELISA assay, and Western blot immunoassay were then used to confirm results. Rheumatoid factor (RF) activity was measured on serum sample by nephelometry (in which a level $>20 \mathrm{IU} / \mathrm{ml}$ was considered positive), and from purified IgMs by ELISA, calculated in UI for $1 \mathrm{ug}$ of IgM.

Results and discussion: A monoclonal B-cell pattern from bone marrow DNA was found in $3 \mathrm{HCV}$ positive patients by GS analysis (Table 1, sample 13 ). The consensus IgVH and VK germline counterparts' region genes have been determined and reported. Peptide masses shared between VH and VL sequences of B-cell receptor and of $\operatorname{IgM}$ peptides were found in all these 3 samples. Identity between B-cell receptor and IgM sequences were corroborated by the identification in both sequences of the same somatic mutations. These data indicated that the cryoprecipitable IgM responsible for the MC syndrome had been produced from the monoclonal B-cell found in the autologous patients. The most probable immunoglobulin family genes for heavy and light chains IgM from other patients had been identified by peptide mass fingerprinting. VH1-69/VK3-20, VH4/VK3-20 and VH3/VK315 gene combinations were predominant. A similar restricted combination of genes was found for $\mathrm{MC}$ and HCV-related NHL DNA $[1,6]$ as well as for IgM recently reported by Damoc E [7]. Intraclonal heterogeneity due to single amino acid difference was also identified in some samples. These finding further corroborated the early demonstration of identity between B cell receptor present on overexpanded B-cell clones and functional region of cryoprecipitated $\operatorname{IgM}$. On the other hand, data support early interpretations that Ig rearrangement and mutations are restricted as the result 
Table 1

Patients' characteristics

\begin{tabular}{|c|c|c|c|c|c|c|c|c|}
\hline & \multirow[t]{2}{*}{ Age/sex } & \multirow[t]{2}{*}{ Diag nosis * } & \multirow{2}{*}{$\begin{array}{l}\text { Bone marrow } \\
\text { B-cell Pattern }\end{array}$} & \multirow{2}{*}{$\begin{array}{c}\text { HCV } \\
\text { genotype }\end{array}$} & \multirow{2}{*}{$\begin{array}{c}\text { Cryoglobulins } \\
\%\end{array}$} & \multirow{2}{*}{$\begin{array}{c}\mathrm{RF} \\
\mathrm{UI} / \mathrm{ml}\end{array}$} & \multicolumn{2}{|c|}{ IgM reactivity } \\
\hline & & & & & & & $\mathrm{HCV}$ & RF \\
\hline 1. & $60 / \mathrm{M}$ & MC and Lymphoplasmacytoid Lymphoma & Monoclonal & $1 \mathrm{~b}$ & 40 & 216,000 & NS3 & ++++ \\
\hline 2. & $57 / \mathrm{F}$ & $\mathrm{MC}$ & Monoclonal & $1 b$ & 19.3 & 107 & NS3 & + \\
\hline 3. & $24 / F$ & MC and Lymphoplasmacytoid Lymphoma & Monoclonal & $2 b$ & 8 & 218 & - & + \\
\hline 4. & $65 / \mathrm{F}$ & MC & Oligoclonal & $1 b$ & 20 & 400 & NS3 & + \\
\hline 5. & $65 / \mathrm{F}$ & MC & Oligoclonal & $1 b$ & 45 & 819 & NS3 & ++ \\
\hline 6. & $70 / \mathrm{M}$ & MC & Oligoclonal & $1 b$ & 19 & 2,410 & NS3 & + \\
\hline 7. & $74 / \mathrm{F}$ & MC & Polyclonal & Positive nd & 40 & 12,600 & NS3 & + \\
\hline 8. & $45 / \mathrm{F}$ & MC & Oligoclonal & Positive nd & 9 & 3,910 & - & +++ \\
\hline 9. & $60 / \mathrm{M}$ & MC & Oligoclonal & $2 \mathrm{a} / 2 \mathrm{c}$ & 7 & 254 & - & ++ \\
\hline 10. & $74 / \mathrm{M}$ & MC and Gastric MALT Lymphoma & Polyclonal & Positive nd & 4 & nd & - & + \\
\hline 11. & $56 / \mathrm{F}$ & $\mathrm{MC}$ & Polyclonal & $2 \mathrm{c}$ & 9 & 801 & - & ++++ \\
\hline 12. & $62 / \mathrm{F}$ & $\mathrm{MC}$ & Oligoclonal & $1 b$ & 9.2 & 3,710 & - & +++ \\
\hline 13. & $73 / \mathrm{M}$ & $\mathrm{MC}$ & Polyclonal & $1 b$ & 1 & 2,580 & - & ++ \\
\hline 14. & $59 / \mathrm{F}$ & $\mathrm{MC}$ & Polyclonal & Positive nd & 4 & 125 & - & ++ \\
\hline 15. & $40 / \mathrm{F}$ & $\mathrm{MC}$ & Oligoclonal & $2 a / 2 c$ & 5 & 382 & - & + \\
\hline 16. & 48/M & $\mathrm{MC}$ & Polyclonal & $1 b$ & 6 & 136 & - & ++ \\
\hline 17. & $75 / \mathrm{F}$ & MC and nodal diffuse large cell lymphoma & Polyclonal & $2 \mathrm{a} / 2 \mathrm{c}$ & 2 & nd & - & ++ \\
\hline 18. & $68 / \mathrm{F}$ & MC in chronic hepatitis B virus infection & Polyclonal & Negative & 4 & nd & - & + \\
\hline 19. & $50 / \mathrm{F}$ & $\mathrm{MC}$ & Oligoclonal & Negative & 3.5 & 150 & - & + \\
\hline 20. & $73 / \mathrm{F}$ & Sjogren'syndrome & Polyclonal & Negative & 1 & 118 & - & + \\
\hline 21. & $47 / F$ & Sjogren'syndrome and Salivary Lymphoma & Polyclonal & Negative & 19 & 4,137 & - & +++ \\
\hline 22. & $68 / \mathrm{F}$ & Sjogren'syndrome & Polyclonal & Negative & 11 & nd & - & +++ \\
\hline 23. & $55 / \mathrm{F}$ & MC in Systemic Lupus Erythematosus & Polyclonal & Negative & 0.5 & 313 & - & ++ \\
\hline 24. & $50 / \mathrm{M}$ & $\mathrm{MC}$ & Polyclonal & Negative & 4 & 30 & - & ++ \\
\hline
\end{tabular}

* =bone marrow was only involved by lymphoma in patient 1 and 3 ; $\mathrm{MC}=$ mixed cryoglobulinemia syndrome;

$\mathrm{RF}$ was determined in UI/ for $1 \mathrm{ug}$ of $\mathrm{IgM}$ as $++++\geqslant$ of $200 \mathrm{UI} / \mathrm{ug} ;+++\geqslant 100 \mathrm{UI} / \mathrm{ug} ;++$ range $\geqslant 12 \mathrm{UI} / \mathrm{ug}$ and $+<12 \mathrm{UI} / \mathrm{ug}$.

of a selective stimulation of specific IgR during the MC progression [1-6]. The demonstration that the cryoprecipitable IgM shares the same clonal origin as the lymphoproliferative process makes $\operatorname{IgM}$ a direct and powerful immunological tool for identification of the antigenic determinant that sustains these lymphoproliferations.

ImmunoAssay indicated that $\operatorname{IgM}$ was directed against NS3 proteins in 6 cases, patients 1-2 and 4-7, (Table 1). Patients showing reactivity to NS3 were all $\mathrm{HCV}$-positive and were infected with HCV genotype $1 \mathrm{~b}$ in 5 cases (sample 7 was not typified). In the remaining HCV-positive patients, the genotype was $1 \mathrm{~b}(3$ cases), $2 \mathrm{a} / 2 \mathrm{c}$ ( 3 cases), $2 \mathrm{~b}$ ( 1 case) and $2 \mathrm{c}$ ( 1 case); 4 samples were not typified. Ig region gene in NS3 reactive sample were preferentially VH1-69, and VK3-20 or VK3-15. IgM reactivity against NS3 protein was confirmed by ELISA and WB. All IgM samples demonstrated a concomitant RF activity. Overall, these data suggest that NS3 protein may be the triggering antigen for HCV-related IgMK lymphoproliferations, at least in a relevant proportion of cases. NS3-reactive cryoprecipitable IgM present also a RF reactivity, finding also supported by Sung et al. [8] which described an IgM produced by an $\mathrm{HCV}$-infected $\mathrm{B}$ cell line reactive against NS3 and IgG-NS3 complex. Further studies are however necessary to ascertain whether NS3 share an antigenic epitope with $\mathrm{IgG}$ or NS3 acts directly in assembling the cryoprecipitable complex. Concerning lymphomagenesis, data are consistent with an indirect role of $\mathrm{HCV}$ as an exogenous trigger. However, since infection of a fraction of B cells appears also likely in some NHL $[3,8]$ (in this study, case 1 was infected though with less than 1 viral genome/cell), the possibility that the virus could explain also a direct role in lymphomagenesis, possibly by means of hit-and run mechanisms [9] may also be considered. In agreement with these conclusions, a proposal for similar therapeutic treatments for patients with low-grade $\mathrm{HCV}$-associated NHL and type II MC may be suggested. In this contest, treatment with interferon and rituximab [10] an antiCD20 B cells monoclonal antibody, proved effective.

\section{References}

[1] V. De Re, S. De Vita, A. Marzotto et al., Sequence analysis of the immunoglobulin antigen receptor of hepatitis $C$ virusassociated non-Hodgkin lymphomas suggests that the malignant cells are derived from the rheumatoid factor-producing 
cells that occur mainly in type II cryoglobulinemia, Blood 96 (2000), 3578-3584.

[2] V. De Re, S. De Vita, A. Marzotto et al., Pre-malignant and malignant lymphoproliferations in an $\mathrm{HCV}$-infected type II mixed cryoglobulinemic patient are sequential phases of an antigen-driven pathological process, Int J Cancer 87 (2000), 211-216.

[3] S. De Vita, V. De Re, D.F. Sansonno et al., Lack of HCV infection in malignant cells refutes the hypothesis of a direct transforming action of the virus in the pathogenesis of $\mathrm{HCV}$ associated B-cell NHLs, Tumori 88 (2002), 400-406.

[4] D. Sansonno, G. Lauletta, V. De Re et al., Intrahepatic B cell clonal expansions and extrahepatic manifestations of chronic HCV infection, Eur J Immunol 34 (2004), 126-136.

[5] S. De Vita, V. De Re, S. Gasparotto et al., Oligoclonal nonneoplastic B cell expansion is the key feature of type II mixed cryoglobulinemia: clinical and molecular findings do not support a bone marrow pathologic diagnosis of indolent B cell lymphoma, Arthritis Rheum 43 (2000), 94-102.

[6] V. De Re, S. De Vita, D. Gasparotto et al., Salivary gland B cell lymphoproliferative disorders in Sjogren's syndrome present a restricted use of antigen receptor gene segments similar to those used by hepatitis C virus-associated non-Hodgkins's lymphomas, Eur J Immunol 32 (2002), 903-910.

[7] E.F. Damoc, N.F. Youhnovski, D.F. Crettaz, J.D.F. Tissot and M. Przybylski, High resolution proteome analysis of cryoglobulins using Fourier transform-ion cyclotron resonance mass spectrometry, Proteomics 3 (2003), 425-433.

[8] V.M. Sung, S. Shimodaira, A.L. Doughty et al., Establishment of B-cell lymphoma cell lines persistently infected with hepatitis $\mathrm{C}$ virus in vivo and in vitro: the apoptotic effects of virus infection, J Virol 77 (2003), 2134-2146.

[9] K. Machida, K.T. Cheng, V.M. Sung et al., Hepatitis C virus induces a mutator phenotype: Enhanced mutations of immunoglobulin and protooncogenes, Proc Natl Acad Sci USA 101 (2004), 4262-4267.

[10] D. Sansonno, V. De Re, G. Lauletta et al., Monoclonal antibody treatment of mixed cryoglobulinemia resistant to interferon alpha with an anti-CD20, Blood 101 (2003), 3818-3826.

\section{[10.40-11.00]}

Evaluation of the biological activity of aurexis ${ }^{\circledR}$, a Humanized $\mathrm{mAb}$ for the first-line treatment of severe $S$. aureus infections in combination with antibiotics

Jeff T. Hutchins, Li Zhang and Joseph M. Patti Inhibitex, Inc. Alpharetta, GA, USA

In preclinical studies, a single administration of Aurexis ${ }^{\circledR}$ (tefibazumab) significantly protected against an IV challenge with a methicillin-resistant $S$. aureus (MRSA) strain in both murine septicemia and rabbit infective endocarditis (IE) models. Moreover, Aurexis ${ }^{\circledR}$ enhanced the therapeutic efficacy of vancomycin therapy in a model of established IE. The biological activity of Aurexis ${ }^{\circledR}$ has been purported to be two-fold; inhibition of the binding of the $S$. aureus MSCRAMM ${ }^{\circledR}$ protein clumping factor (ClfA) to the fibrinogen and immune clearance by opsonophagocytosis. This re- port begins to delineate the mechanism of action for Aurexis ${ }^{\circledR}$. To investigate the contribution of the inhibition of fibrinogen binding to the efficacy, sitespecific mutations were introduced into the variable light chain CDR3 domain to generate mutated versions of Aurexis ${ }^{\circledR}$ that possess reduced inhibitory activity. In addition, $\mathrm{a} \mathrm{mAb}$ that exhibits high affinity for ClfA, but possesses no detectable inhibitory activity was produced. The biological activity of these mAbs was evaluated in an inhibition assay and in an in vitro phagocytosis assay. The protective efficacy of these recombinant antibodies was also analyzed in preclinical animal models of MRSA infection. Taken together, the data from the in vitro and in vivo experiments clearly support the prevailing concept that inhibition of binding and opsonophagocytosis together contribute to the overall biological activity of Aurexis ${ }^{\circledR}$.

\section{[11.00-11.20]}

Identification of an immune receptor on primary $B$ cell leukaemia cells down-regulating a Th1 immune response

Kathy Bowdish

Alexion Technologies Inc., USA

Abstract not received

\section{[11.20-11.50]}

[Keynote Lecture]

Phage display- generated recombinant Fabs for the study of human antiviral immunoglobulin subpopulations

Roberto Burioni ${ }^{1}$, Nicasio Mancini ${ }^{1,2}$, Silvia

Carletti $^{2}$, Mario Perotti ${ }^{2}$, Antonella Grieco ${ }^{1}$, Eleonora Belardinelli ${ }^{1}$, Martina Mammarella ${ }^{1}$, Michela

Sampaolo1 Filippo Canducci ${ }^{2}$ and Massimo Clementi $^{2}$

${ }^{1}$ Università di Ancona, Milan, Italy

${ }^{2}$ Università “Vita e Salute” San Raffaele, Milan, Italy

The tendency of some viral infections toward chronicity with persistent viral replication in spite of a strong antibody response suggests that the cases of persistent viral infections the host immune response is unable to effectively tackle the infection. For example, the protection afforded to the host by the presence of antibodies directed against hepatitis $\mathrm{C}$ virus (HCV) envelope glycoproteins (E1 and E2) is still unclear, and clinical data are conflicting, even if in some controlled study the protective effect of passive immunotherapy was clearly shown. 
Recent data obtained by our group by dissecting the human humoral immunoresponse by molecular cloning of the antibody repertoire of a persistently infected patient in phage display combinatorial vectors shed some lights on the role of antibodies in the virus-host interplay, suggesting that a remarkable part of the humoral immunoresponse seems to be not necessarily beneficial to the host providing a molecular basis to the lack of consistent protection provided by the anti-HCV response. A major advance in understanding the role played by antibodies recognizing the different epitopes would be to correlate their presence with viremia levels, disease progression, therapy effectiveness or other clinical data. Unfortunately the major B epitopes recognized by the human immune system and identified by our group are conformational, and can not reproduced by peptide scan for an ELISA quantitation.

In this presentation we describe the development and the validation of a novel strategy for the in vitro quantitation of human antibody subpopulations recognizing the B epitopes present on the surface of HCV/E2. We developed a genetic labeling strategy of human recombinant Fabs allowing us to design a competition ELISA test. We furthermore validated our quantitation method constructing mocked serum specimens containing known amount of IgG1 antibody recognizing a given epitope and showing a good correlation of the inhibition of the binding of labeled Fabs with the amount of antibody actually contained in the serum. Finally, we defined a new measure for the amount of antibodies against each single HCV/E2 B epitope present in the serum of the patient named Fab Inhibition Titer (FIT) that we applied preliminarily to a set of $\mathrm{HCV}$ positive sera with different viremia level.

In conclusion, the FIT test described in this paper is a fast and high-throughput assay able to measure the amount of antibodies directed against conformational epitopes present on the E2 surface, and it can be adapted to any other viral or bacterial pathogen. In this particular case, the antibody response against E2 glycoprotein, considered the most important viral structure interacting with cellular targets in the initiation of $\mathrm{HCV}$ infection, can now be dissected and studied to a deeper level, obtaining new information on interaction between antibodies and virus.

[11.50-12.30]

[Keynote Lecture]

The future of antibodies versus small molecule pharmaceuticals

Jim W. Larrick

Panorama Research Institute, Mountain View, CA, USA

Abstract not received. 